\title{
An exceptional horizontal gene transfer in plastids: gene replacement by a distant bacterial paralog and evidence that haptophyte and cryptophyte plastids are sisters Danny W Rice and Jeffrey D Palmer*
}

Address: Department of Biology, Indiana University, Bloomington, IN 47405, USA

Email: Danny W Rice - dwrice@indiana.edu; Jeffrey D Palmer* - jpalmer@bio.indiana.edu

* Corresponding author

Published: 06 September 2006

BMC Biology 2006, 4:3I doi:|0.|I86/|74|-7007-4-3।
Received: 05 June 2006

Accepted: 06 September 2006

This article is available from: http://www.biomedcentral.com/I74I-7007/4/3 I

(c) 2006 Rice and Palmer; licensee BioMed Central Ltd.

This is an Open Access article distributed under the terms of the Creative Commons Attribution License (http://creativecommons.org/licenses/by/2.0), which permits unrestricted use, distribution, and reproduction in any medium, provided the original work is properly cited.

\begin{abstract}
Background: Horizontal gene transfer (HGT) to the plant mitochondrial genome has recently been shown to occur at a surprisingly high rate; however, little evidence has been found for HGT to the plastid genome, despite extensive sequencing. In this study, we analyzed all genes from sequenced plastid genomes to unearth any neglected cases of HGT and to obtain a measure of the overall extent of HGT to the plastid.
\end{abstract}

Results: Although several genes gave strongly supported conflicting trees under certain conditions, we are confident of HGT in only a single case beyond the rubisco HGT already reported. Most of the conflicts involved near neighbors connected by long branches (e.g. red algae and their secondary hosts), where phylogenetic methods are prone to mislead. However, three genes - clpP, $y c f 2$, and $r p l 36$ - provided strong support for taxa moving far from their organismal position. Further taxon sampling of $c l p P$ and $y c f 2$ resulted in rejection of HGT due to long-branch attraction and a serious error in the published plastid genome sequence of Oenothera elata, respectively. A single new case, a bacterial $r p / 36$ gene transferred into the ancestor of the cryptophyte and haptophyte plastids, appears to be a true HGT event. Interestingly, this rpl36 gene is a distantly related paralog of the rp/36 type found in other plastids and most eubacteria. Moreover, the transferred gene has physically replaced the native rpl36 gene, yet flanking genes and intergenic regions show no sign of HGT. This suggests that gene replacement somehow occurred by recombination at the very ends of $r p / 36$, without the level and length of similarity normally expected to support recombination.

Conclusion: The rp/36 HGT discovered in this study is of considerable interest in terms of both molecular mechanism and phylogeny. The plastid acquisition of a bacterial rpl36 gene via HGT provides the first strong evidence for a sister-group relationship between haptophyte and cryptophyte plastids to the exclusion of heterokont and alveolate plastids. Moreover, the bacterial gene has replaced the native plastid rp/36 gene by an uncertain mechanism that appears inconsistent with existing models for the recombinational basis of gene conversion. 


\section{Background}

Unlike the dynamic mitochondrial genome of flowering plants, which frequently incorporates plastid and nuclear sequences via intracellular gene transfer [1-3], the plastid genome is highly resistant to the uptake of intracellular DNA [4,5]. Recently, a large number of discoveries of HGT involving mitochondrial genes of land plants have been reported [6-15]. Most, if not all of these transfers seem to be the result of a gene being transferred from the mitochondrial genome of one species to that of another. No analogous case of plastid-to-plastid transfer has been reported, but these mitochondrial discoveries recommend a thorough assessment of plastid HGT.

To date, only a single non-intron example of HGT to the plastid has been found. This is the ancient transfer of the rubisco operon $(r b c L$ and $r b c S$ ) from a proteobacterium into the common ancestor of red algal plastids and their secondary derivatives [16], a case that is revisited in this study. In contrast to transfers of constituent genes, acquisition of new introns may be relatively common in plastids [17-25], based on their disparate phylogenetic distribution among plastid genomes, especially in green algae, and the fact that some introns are mobile elements.

The evidence found thus far for HGT to the plastid proceeded from studies of a particular gene or intron. To quantify the overall extent of HGT in plastid genomes, we searched exhaustively for HGT among the 42 sequenced plastid genomes available when this study began. Our search relied primarily on phylogenetic analyses, but also involved scrutiny of each potential case (including generation of new gene sequences from phylogenetically relevant taxa) to rule out artifacts and various types of homoplasy.

\section{Results}

Of the 204 protein genes present in four or more of the 42 examined plastid genomes, 34 produced maximum likelihood (ML) trees that had at least one node that conflicted (see Methods) with the reference plastid tree (Additional File 1), with bootstrap proportion (BP) $\geq 80 \%$. Fifteen had conflicts with $\mathrm{BP} \geq 90 \%$, and 11 had conflicts with BP $\geq 95 \%$. Thirteen of the genes with $\mathrm{BP} \geq 80 \%$ involved rhodophyte/Odontella/Guillardia relationships, eight involved conflicts within the four grass taxa, and the rest were various other conflicts. In eight trees, multiple nodes had well-supported conflicts. Usually these pointed to a single rearrangement in the tree, but three trees had wellsupported conflicts in different regions of the tree.

After closer analysis, in some cases requiring the generation of additional sequences for key taxa, none of these conflicts proved to be strong cases of HGT. Ironically, one case that was not detected by this phylogenetic filter involves a very short gene that nonetheless offers strong support for a bacterium-to-plastid HGT.

\section{HGT of rpl36}

The Guillardia theta rpl36 gene is very divergent from the rpl36 genes present in the surveyed plastid genomes and in cyanobacteria. In trees, it branches with a paralogous rpl36 type with strong support regardless of the phylogenetic method used (Figure 1). Here we refer to the type found in Guillardia as rpl36-c (for cryptophyte), and the type found in most plastids and most cyanobacteria as rpl36-p (for plastid). The 144-bp-long Guillardia gene shares, with all rpl36-c genes relative to rpl36-p, three indels (insertions of one and six amino acids, and a deletion of three amino acids), as well as an overall aminoacid and nucleotide similarity (Figure 1 and Additional File 2). Guillardia rpl36-c has a 7 amino-acid 3' extension present in 18 gamma-proteobacterial species, in the planctomycete Rhodopirellula baltica, and in the cyanobacterium Crocosphaera watsonii (Additional File 2). The rpl36 HGT was not detected by our initial phylogenetic filter because our trees sampled only plastid-containing taxa, and this gene is too short to give strongly supported groupings within the plastids (Additional File 3). We detected this conflict only after building trees containing a broader sampling of $r p l 36$ genes.

In addition to the plastids and cyanobacteria, rpl36-p is found across many groups of bacteria, including diverse proteobacteria, and in fungal nuclear genes targeted to the mitochondrion. Most gamma-proteobacteria and a few beta-proteobacteria and actinobacteria contain both forms of the rpl36 gene (e.g. Figure 1). Crocosphaera watsonii has an rpl36-p with a frame-shift insertion near the 5 ' end, suggesting that it has been functionally replaced by a horizontally transferred rpl36-c.

The rpl36 gene is located between $\sec Y$ and $r p s 13$ in all six sequenced plastid genomes from red algae and their secondary photosynthetic derivatives, including Guillardia theta (Additional File 2). This is within a larger syntenic group of 22 genes conserved in the red algal plastids and diverse bacterial lineages. None of the rpl36-c genes in bacteria are adjacent to $\sec Y$ or $r p s 13$, nor are any located within the larger syntenic region.

To identify the approximate time/phylogenetic boundary of transfer and to confirm the validity of the Guillardia gene, we sequenced rpl36 from three additional, diverse [26] cryptophytes: Hanusia phi, Chroomonas mesostigmatica, and Cryptomonas tetrapyrenoidosa. Using PCR, we isolated only rpl36-c from all three cryptophytes (and only rpl36-c was found in the unpublished plastid genome sequence of the cryptophyte Rhodomonas salina CCMP1319; H. Khan and J. Archibald, personal commu- 


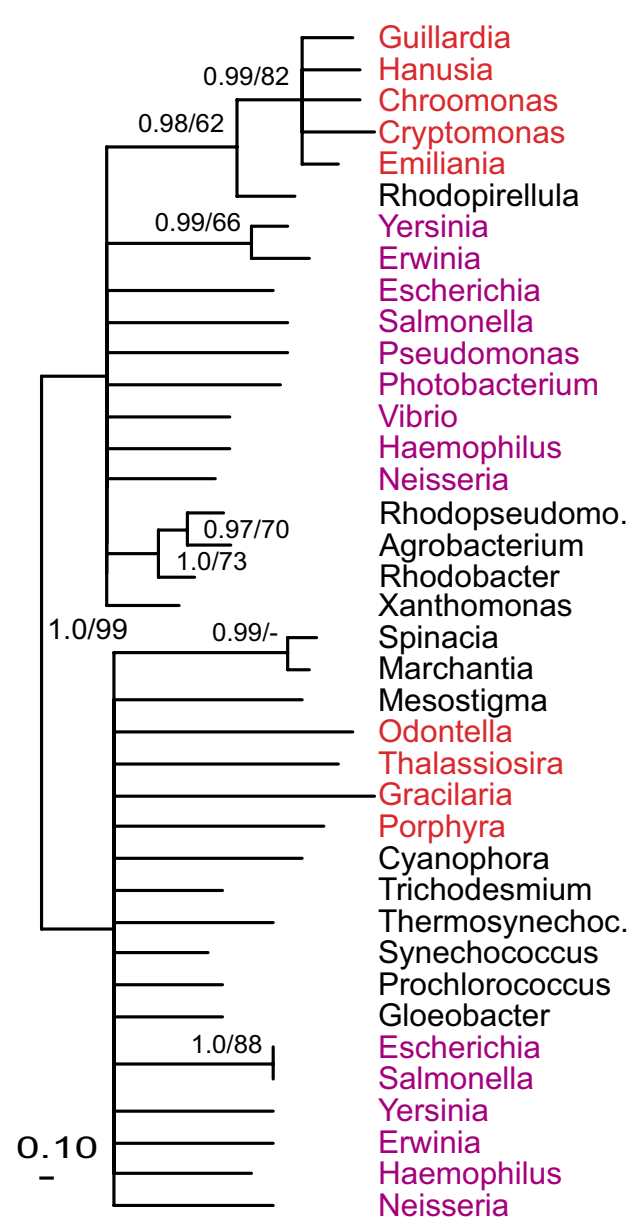

Figure I

rpl36 tree and alignment. The M3 codon model in MrBayes was used to calculate the tree using the alignment shown. Nodes with posterior probability $<0.95$ are collapsed. Posterior probabilities (left) and PROML BP values $>50 \%$ (right) are shown on the remaining nodes. The PROML bootstraps were run with four rate categories (estimated with PUZZLE) and global rearrangements. Nucleotide and amino-acid based ML analyses using PAUP* and MrBayes also gave $100 \%$ support for the division between the c-type and p-type rpl36 genes. This support is maintained when all positions containing gaps are removed. Because the 3' extension unique to some c-type rp/36 genes (see Additional File 2) was excluded from this phylogenetic analysis, it is not shown in the alignment. In the alignment, each base is colored according to the key. Taxa in red include the red algae and their secondary plastid containing relatives. A subset of the many proteobacterial species which contain both the $p-$ type and c-type genes is shown in purple. The p-type Pseudomonas, Photobacterium, and Vibrio genes are not shown here.

nication). These genes possess high sequence similarity (nucleotide identity between $79 \%$ and $96 \%$ ) to $r p l 36$ of Guillardia theta. We obtained high-quality sequence for a region comprising all of $r p l 36$, both of its flanking spacers, 219 bp at the 3' end of $\sec Y$, and $300 \mathrm{bp}$ at the $5^{\prime}$ end of rps13 (Figure 1 and Additional File 2).

The plastid genome of the haptophyte Emiliania huxleyi, which was sequenced too recently [27] to be included in this study, also contains rpl36-c in place of rpl36-p (Figure 1 and Additional File 2). Emiliania rpl36 shares the c-type indels and 3' extension with the cryptophyte rpl36 genes and contains no additional indels over its entire length. Its amino-acid identities to the cryptophyte rpl36-c genes range from 85 to $90 \%$, and its nucleotide identities range from 72 to $79 \%$. It too is located between $\sec Y$ and $r p s 13$, with $5^{\prime}$ and 3 ' intergenic spacers of length 139 bp and 14 $\mathrm{bp}$, respectively. The Emiliania sequence groups as sister to the cryptophyte rpl36-c genes with good support (Figure 1). In addition, an EST sequence http:// tbestdb.bcm.umontreal.ca from the dinoflagellate Karlodinium micrum, which possesses a tertiary plastid of hapto- 
phyte origin [28-30], also contains the rpl36-c gene. Furthermore, the Karlodinium sequence is sister to the Emiliania sequence in phylogenetic analyses (data not shown, but see Additional File 2).

\section{The clpP conflict}

In what follows, we describe and discuss genes that initially gave conflicting trees with relatively high bootstrap support, but which for various reasons were either strongly rejected or brought into question as potential horizontal transfers.

The $c l p P$ gene from Oenothera elata is highly divergent. With the taxon sampling used in this study (Additional File 1), it branches, with $84 \%$ BP (see Figure 2 legend), as the sister to the grasses, which are also a long-branched group (Figure 2A). Suspecting long-branch attraction (LBA), we obtained more genes (provided by L. Goertzen and C. Long) from the order Myrtales, to which Oenothera belongs (including Clarkia, Fuschia, Eucalyptus, Punica, Callistemon, and Oenothera organensis) and from other non-grass monocots (including Acorus and Flagellaria) to test if the grouping with the grasses was an artifact. The resulting tree (Figure $2 \mathrm{~B}$ ) strongly suggests that the original result was an LBA artifact, as Oenothera goes within the Myrtales (and within its family Onagraceae) with the better sampling.

\section{The ycf2 conflict}

The published Oenothera elata $y c f 2$ gene branched as sister to the asterids Atropa and Nicotiana with BP of $100 \%$, instead of with other rosid sequences (e.g. Arabidopsis and Lotus). To verify this, we sequenced $\gamma c f 2$ from a number of diverse Myrtales including O. elata itself. This new Oenothera sequence did not match the published $O$. elata plastid genome sequence [31], several regions of which (up to $1.5 \mathrm{~kb}$ in length) have $100 \%$ sequence identity with the Nicotiana $y c f 2$ gene (Figure 3A). These cover regions that have long insertions in our $O$. elata sequence but that are missing in the $O$. elata genome sequence. This latter sequence also contains insertions shared with Nicotiana but not with our $O$. elata $y c f 2$ sequence. Regions in the published sequence that do match our sequence appear to have single base errors, given that our $O$. biennis sequence is more similar to our $O$. elata sequence than is the published sequence in these regions (Figure 3A). Although

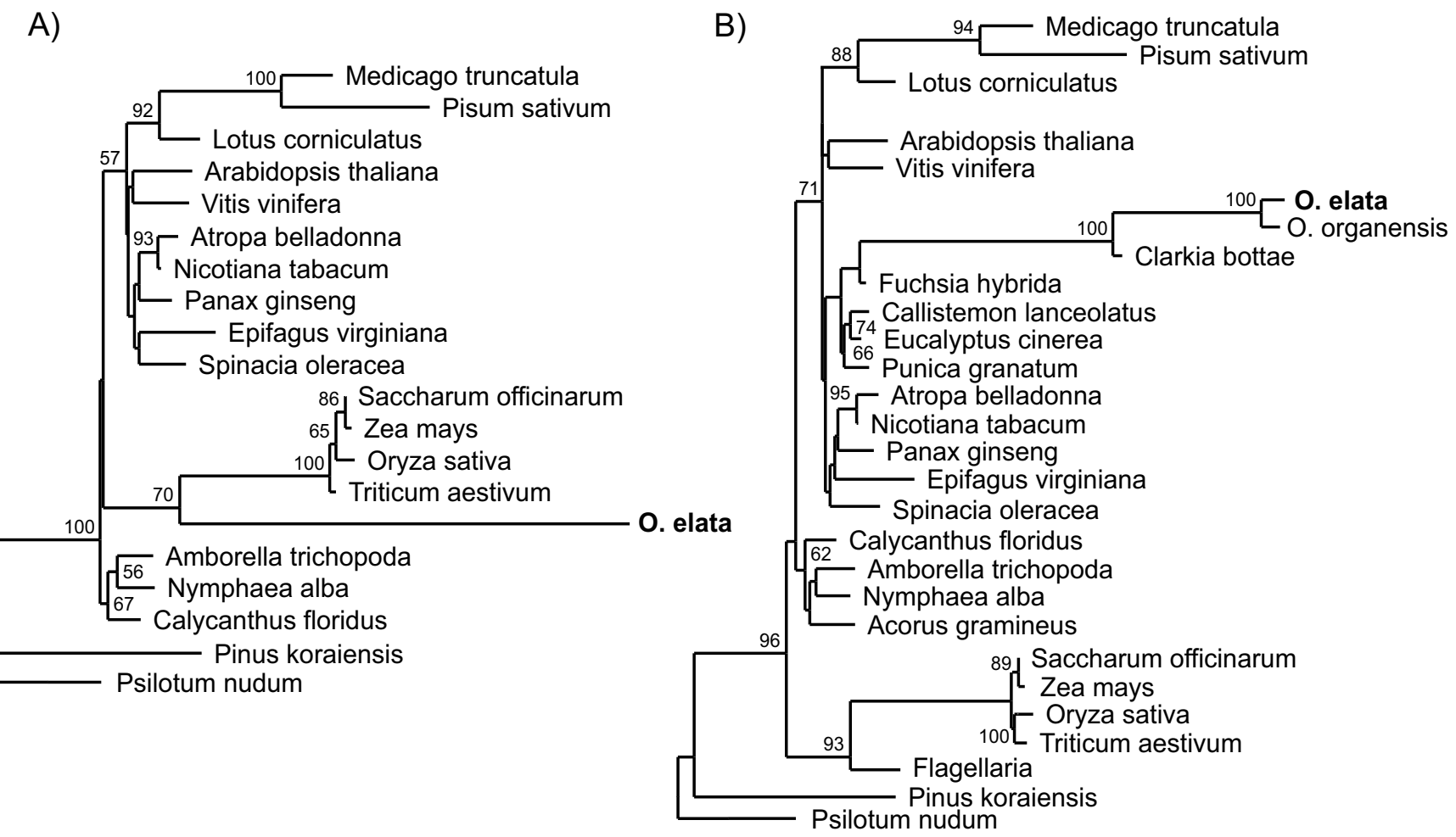

Figure 2

clpP phylogeny before and after taxon addition. ML analysis was performed on an all-position nucleotide alignment using PAUP* as described in Methods with the TVM+G model used for both trees. A 60-bp 3' extension with questionable homology across taxa was removed in this analysis but was included in the original analysis. This is probably responsible for the change in the BP from $84 \%$ originally (not shown) to $70 \%$ here for Oenothera going with grasses in tree (A). Bootstrap values $<50 \%$ are not shown. (A) Original taxon sampling; (B) after new taxa added. 


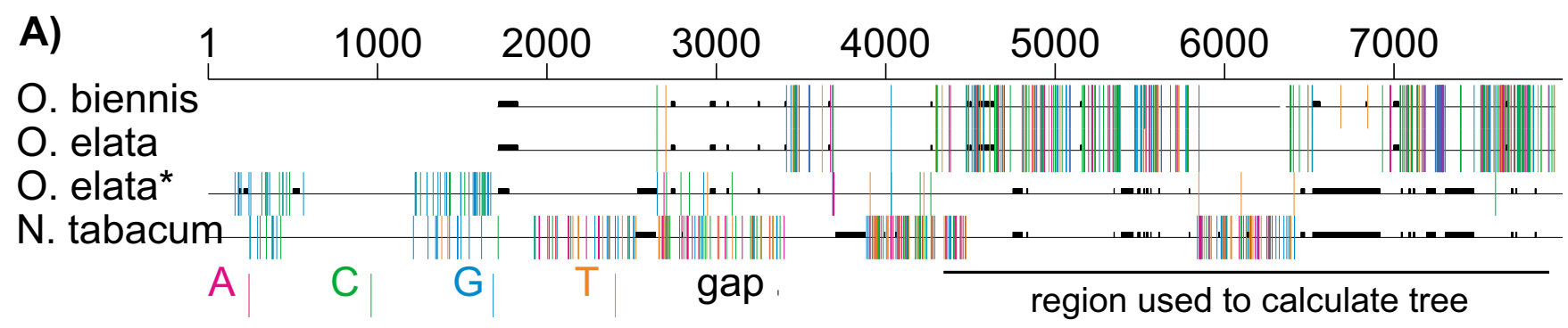

B)

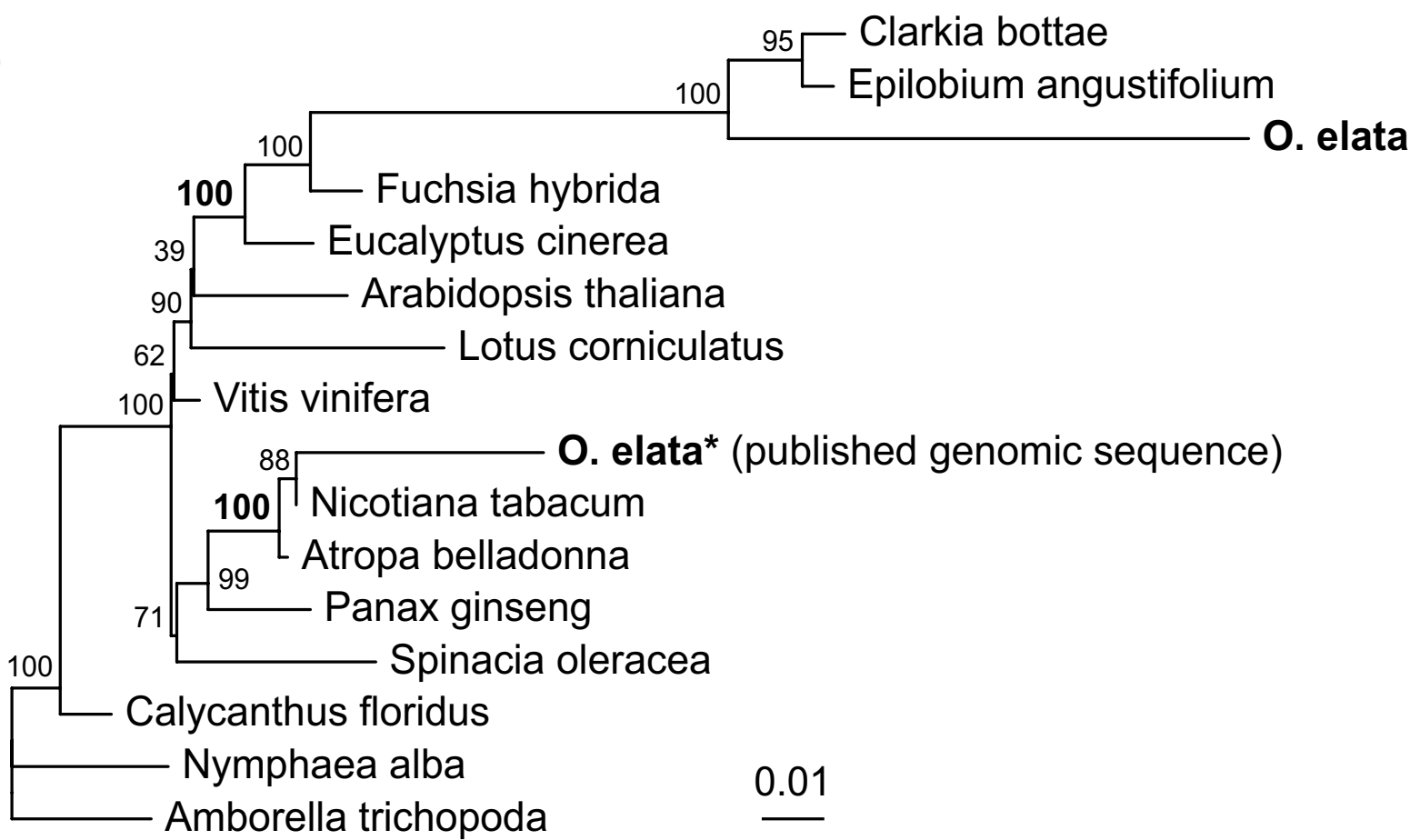

Figure 3

Error in the published Oenothera elata ycf2. (A) Alignment of ycf2 nucleotide sequences: The top two sequences, Oenothera biennis and $O$. elata, were sequenced as part of this study. We did not sequence the first $\sim 1600 \mathrm{bp}$. The bottom two sequences correspond to the published Oenothera elata and Nicotiana tabacum sequences. The bottom three sequences were used to determine a consensus base at each position, and positions that did not match this consensus are colored as denoted in the key. (B) All codon position ML tree using the TVM+G model in PAUP* with 100 bootstrap replicates. Only the 3' region of ycf2 starting at position 4023 of the published Oenothera sequence [3I] was obtained for the four Myrtales taxa (Eucalyptus, Fuchsia, Clarkia and Epilobium) and the analysis was performed using this region of aligned positions as indicated in (A). Within this region, gappy positions were removed prior to phylogenetic analysis, which resulted in 2567 positions. When the entire gene was used with the published Oenothera sequence excluded, the topology was the same except that the Lotus and Arabidopsis branches were switched. When the published Oenothera is included in the full-length analysis, its strong chimerism pulled Clarkia, Epilobium and our elata sequence into an artifactual clade with the published elata gene at the base of the Solanaceae.

very divergent, the new $O$. elata sequence branches in the expected position with other Myrtales (Figure 3B). We strongly suspect that this conflict is due to extensive error in the $y c f 2$ region of the published O. elata genome [31], perhaps due to inadvertent incorporation of Nicotiana sequence during genome assembly (see Additional File 4). 


\section{The rhodophyte/chromalveolate conflicts}

The chromalveolates [Guillardia (cryptophytes), Odontella (heterokonts), and apicomplexans in our sampling] are a putatively monophyletic group that, with respect to plastid phylogeny, branch within the red algae, with the Cyanidiales being sister to the Porphyra/Gracilaria/ chromalveolate clade (Additional File 1). Relationships among the different chromalveolate groups are not well established [32], but the consensus topology provides a reasonable working hypothesis. Most of the conflicts that we found relative to this topology involve Guillardia and/ or Odontella branching as sister to all the rhodophytes instead of as sister to Porphyra/Gracilaria. Although some of these conflicts could in principle be true cases of HGT, the combination of long branches and near-neighbor exchanges makes these conflicts suspect, even given high bootstrap support. For some of these we have seen evidence (assuming our plastid tree is correct) of codonusage bias. For example, the $p s b B$ gene tree goes from $100 \%$ BP for rhodophytes being monophyletic to the exclusion of Guillardia and Odontella, using all three codon positions, to weak support for a topological change to the consensus tree when only second positions are used. First and second positions together still give strong support for the conflicting tree, indicating that first positions may also contribute a significant bias for $p s b B$ [33].

Another well-supported conflict, psbA, is discussed in Additional File 5. The remaining genes that had conflicts supported by BP of $80 \%$ or higher are $p s b C$, atpH, psaK, dnaK, atpF, atpB, rpl31, ycf4, ycf17, ycf45, and $\gamma c f 37$. As above, we could not reject the conflicts outright, but we could show weakened support or induce topological changes with alternative data filtering such as using second codon positions alone or amino acids. In no case did we observe any telltale signals such as uniquely shared indels in the conflicting clades.

\section{The grass conflicts}

Eight gene trees conflicted with the consensus tree (Additional File 1) with respect to relationships among the four grasses examined. Four gene trees (atpI, $p s b H$, atpF, rpl16) supported the monophyly of Triticum/Saccharum/Zea, while the other four ( $r p l 22, n d h D, p s a A, p s b A)$ supported monophyly of Oryza/Saccharum/Zea. In all cases there is a long branch leading to the grasses, which reflects both the lack of any close outgroups (no other monocots were included) and the well-established rapid evolution of the chloroplast genome in the stem group leading to grasses (Stefanovic et al [34] and references therein). We hypothesize that all eight gene trees whose within-grass topologies conflicted with the consensus topology reflect spurious results stemming from the lack of close outgroups to the grass sequences. To test this hypothesis, we reanalyzed the two genes (atpI and $p s b H)$ that showed the highest level of conflict ( $\mathrm{BP}=96 \%)$, using all other monocot sequences available (from Sorghum, Hordeum, Phyllostachys, Typha, Yucca, Phalaenopsis, and Acorus) for these two genes. With this improved sample, the atpI phylogeny was no longer in significant conflict with the organismal tree; instead, relationships among Triticum, Oryza, and Saccharum/Zea were entirely unresolved under an all nucleotide position ML model (Additional File 6). For $p s b H$, however, the situation did not change markedly; we obtained a BP of $91 \%$ for Oryza being sister to Triticum/ Saccharum/Zea, but this corresponds to only three parsimony informative characters. Such a small number of informative characters could easily be homoplasious, and better taxon sampling is required to resolve these conflicts firmly.

\section{Other conflicts}

The remaining conflicts with $\mathrm{BP} \geq 80 \%$ were all brought into question using alternative phylogenetic analyses that led to reduction of bootstrap support or topology change. All of these involved conflicts with branches that are near each other in the consensus tree.

\section{rRNA and tRNA genes}

The small and large subunit ribosomal RNA genes had strong support for the euglenids going outside the green algae (sister to red/green algae for $16 \mathrm{~S}$ and within the red algae for 23S). Increasing taxon sampling using other available rRNA genes [35] gave a weakly supported placement of the euglenids within the green algae. No clear cases of HGT in tRNA genes were detected, but interpretation of these alignments and trees is difficult, owing to the short length and extensive paralogy of these genes.

\section{Scrutiny of long-branched lineages}

The approach used here is limited by the taxon sampling. Because our initial trees included only plastid genes, we could essentially detect HGT only from one plastid genome to another, but not transfers from other genomes into plastids. We reasoned that transfers of non-plastid genes should normally result in a long branch leading to the donee taxon within the plastid gene trees. A description of our analysis of these long-branch lineages is presented in Additional File 7. No additional cases of HGT were detected in this analysis.

\section{Discussion}

\section{The rpl36 transfer: the chromalveolate hypothesis and algal phylogeny}

The unique, derived presence of the horizontally transferred rpl36-c gene in haptophyte and cryptophyte plastids, but not in heterokont and alveolate plastids, provides the first strong evidence for the "sisterhood" of haptophyte and cryptophyte plastids. The most parsimonious scenario is that the rpl36-c gene was transferred once 
to the ancestral plastid of the haptophytes and cryptophytes after this plastid lineage and the lineage(s) leading to heterokonts and alveolates diverged. Less parsimonious alternative scenarios can be imagined, but the similarity between the haptophyte and cryptophyte rpl36 genes, their position as sister lineages among the rpl36-c genes (Figure 1), and the fact that the transfer appears to have occurred via an improbable recombination event make alternative explanations unlikely.

The haptophytes and heterokonts have been recognized as sister groups based on ultrastructural and pigment similarities [36,37], and named 'chromobiotes'. In addition, phylogenies based on concatenated plastid genes tend to group the haptophytes and heterokonts [38,39] (see Additional File 8 for further discussion of chromalveolate phylogeny). However, on a per-gene basis, the signal is mixed, and nearly half the plastid genes actually group haptophytes and cryptophytes as sisters (Additional File 9 and Additional File 10). The morphological characters linking haptophytes to heterokonts could all be ancestral to the chromophytes (chromobiotes plus cryptophytes) or even the chromalveolates (chromophytes plus alveolates) $[32,40])$ and lost differentially. For example, chlorophyll c3 and autofluorescence of the rear cilium could have been lost in the cryptophytes, and the nucleomorph (present in cryptophytes) could have been lost independently in the haptophytes and heterokonts (it is well established that the nucleomorph has been independently lost in the secondary, green-derived plastid of euglenids). In contrast, the presence of the c-type rpl36 in only haptophytes and cryptophytes cannot be explained by differential loss unless one posits its unlikely insertion via HGT immediately adjacent to, rather than in place of, the ancestral p-type gene. It remains to be seen whether the hypothesis of haptophyte/cryptophyte plastid monophyly is supported or rejected by future phylogenetic analyses involving many more plastid and nuclear genes and/ or taxa from across the chromalveolates. One possibility, which is based on the serial symbiosis models developed by Bachvaroff et al $[38,39]$, is that the cryptophyte and haptophyte plastids, but not their nuclear lineages, will turn out to be sister groups. This would be the case if, say, the cryptophyte plastid was of secondary, red-algal origin and the haptophyte plastid of tertiary, cryptophyte origin. However, a study using six nuclear cytosolic protein genes did group haptophytes and cryptophytes with weak support [41], suggesting that their nuclear genes may also be monophyletic.

\section{The donor of the rpl36-c gene}

rpl36-c was probably transferred to the ancestral plastid of haptophytes and cryptophytes directly from a bacterium rather than from the mitochondrion or nucleus, as there is no evidence of a rpl36-c in these compartments. At the amino-acid level, plastid rpl36-c is most similar to Rhodopirellula baltica. Interestingly, the complete shotgun sequences from two other planctomycetes (Blastopirellula marina and Gemmata obscuriglobus) contain only rpl36-p. Thus, a potential transfer between the Rhodopirellula lineage and the cryptophyte lineage would likely postdate the Rhodopirellula/Blastopirellula/Gemmata divergence. However, a recent HGT from an unknown donor into Rhodopirellula is also possible. On balance, based on the current bacterial sampling, the donor of the plastid rpl36-c was most likely a planctomycete related to Rhodopirellula or a proteobacterium. A cyanobacterium related to Crocosphaera watsonii is a less likely but potential donor since the Crocosphaera branches within the gamma-proteobacterial c-type group (tree not shown; but see Additional File 2 ), but was probably recently acquired from this group via HGT (see Results).

\section{The rpl36 transfer: mechanism and functional consequence}

Because plastid rpl36-c and rpl36-p are both located between secY and rps13 in the same orientation, we suspect that the rpl36 HGT was mediated by homologous recombination. This would be extraordinary, because the Guillardia and Porphyra rpl36 genes are only 49\% identical in nucleotide sequence in non-gap regions. At this level of divergence, homologous recombination is thought to be highly unlikely $[42,43]$. It is implausible that flanking sequence could have been used to initiate gene conversion, as intergenic regions between distant taxa are essentially random, and no bacterial c-type rpl36 genes are flanked by secY and rps13. Additionally, the 3' end of $\sec Y$ and the 5' end of rps13 in Guillardia do not appear to have been replaced by divergent sequence, as they are still highly similar to the red algal and cyanobacterial genes relative to Rhodopirellula and proteobacteria (Additional File 2A). Even the last 30 bases of Guillardia $\sec Y$ have a higher sequence identity to red algal and cyanobacterial homologs than to all other known sequences. As the 3' end of the rps13 first and second position alignment is iteratively removed, Guillardia continues to group with the red algae and cyanobacteria until about $40 \mathrm{bp}$ are left, at which point phylogenetic resolution is lost, owing to relatively high sequence conservation. There is no significant sequence similarity between the Guillardia rpl36 intergenic regions and those from any available c-typecontaining bacteria. In fact, there is only modest conservation among the intergenic regions of the additional cryptophyte genomes we examined. The cryptophyte intergenic spacers $3^{\prime}$ to rpl36 ranged in length from 42 to 53 bp with sequence identities, in non-gap regions, of 68$80 \%$, while the 5 ' spacers ranged in length from 30 to 150 bp with sequence identities of 59-74\%. 
This leads to the hypothesis that recombination may have been initiated by very short regions of conservation between the rpl36-c and rpl36-p genes themselves. Most reported recombination events between bacterial species tend to be among highly similar sequences [44]. However, this may not be entirely due to the level of sequence similarity but also to interspecies barriers, such as mismatch repair [45]. The minimal sequence identity required to initiate recombination varies depending on the system and species being tested, but has been shown to involve 20 or fewer consecutive identical nucleotides for some types of recombination $[46,47]$.

Although the overall similarity between $r p l 36$-c and $r p l 36-$ $\mathrm{p}$ is very low (Figure 1), the $5^{\prime}$ and $3^{\prime}$ ends are more conserved than the rest of the gene. Specifically, the plastid rpl36-c genes share an 8-bp 5' sequence (AGTAAAGT) with all rpl36-p genes in the red algal lineage, as well as with several green algae and land plants and with several rpl36-p and rpl36-c bacterial genes. A less well-conserved 6-bp 3' sequence (CAAGGT) exists in cryptophyte and some alpha-proteobacterial $r p l 36$-c genes and in the rpl36p gene from some cyanobacteria, land plants, green algae, and red algae. This identity extends leftwards by a further 3 bp (CGTCAAGGT) in Cryptomonas, some cyanobacteria, and Odontella. These similarities between the plastidal and bacterial rpl36-c and the rpl36-p genes in the red-plastid lineage are consistent with one or both ends being involved in recombination. A gene replacement along these lines would represent, to our knowledge, an unprecedented recombination event in terms of sequence distance. Although it is conceivable that the rpl36-c and rpl36-p genes involved in this putative recombination some 1 billion years ago shared more sequence similarity than the extant genes, rpl36-c has not diverged greatly among haptophytes and cryptophytes, and rpl36-p is quite conserved among plastids (Figure 1).

This being the case, possible alternatives to recombination, and HGT itself, should be considered, such as convergent evolution. However, even though rpl36 is very short, convergence is unlikely, given the sequence divergence between the $\mathrm{p}$ - and c-type rpl36 genes and that the algal c-type genes emerge as a nested clade from within the larger group of c-type genes (Figure 1). The convergence hypothesis would require a staggering and unprecedented number of convergent events. At the amino-acid level, the Guillardia rpl36 shares 36 identities with Rhodopirellula, but only 13-18 identities with its red-algal relatives. It also shares three gaps and a 7 amino-acid 3' extension with bacterial rpl36-c (Figure 1 and Additional File 2). Although functional convergence does occur in protein genes [48], nothing approaching the extent that must be invoked for $r p l 36$ has been shown. Protein functional convergence usually occurs at very short, key areas of the protein; for example, within active site regions of an enzyme.

In contrast to convergent positive selection for function, GC content can have a dramatic effect on amino-acid and codon usage [49]. However, plastids, including Guillardia and Emiliania, have a low genomic GC content relative to the bacterial genomes of the taxa shown in Figure 1. The rpl36-c genes mirror the genomic GC content. For example, Guillardia rpl36-c is 31\% GC (genome is 33\%) while Rhodopirellula rpl36-c is 54\% (genome is 55\%). In contrast to convergence, these differences in GC content probably account for much of the divergence between the plastid and bacterial rpl36-c genes and make chance convergence much less likely.

rpl36-c almost certainly physically replaced $r p l 36$-p in certain algae; but did it also functionally replace it? The two types are highly divergent from one another (Figure 1 and Additional File 2), and to our knowledge, rpl36-c has never been shown to play the equivalent ribosomal function in any organism. It is therefore conceivable that a nuclear-encoded, plastid-targeted rpl36-p exists in the cryptophytes and haptophytes, while rpl36-c serves some other function. However, upon consideration of the crystal structure of the 50S ribosomal subunit from E. coli [50] it is plausible that rpl36-c could functionally replace rpl36p. First, the amino acids making van der Waals and hydrogen bond contacts with the 23S ribosomal RNA are fairly well conserved between the two rpl36 types (Additional File 2). Second, the region where the 3 amino-acid insertion exists in the c-type makes no intermolecular contacts, but instead protrudes into the solvent where an insertion is unlikely to cause a functional problem. Interestingly, this insertion creates potential $\mathrm{N}$-myristoylation and $\mathrm{N}$ glycosylation sites that could have functional importance. Third, the crystal structure reveals a large empty space, in contact with the C-terminal glycine, that could easily accommodate the 7 amino-acid C-terminal extension in rpl36-c. Thus, it is reasonable to expect that rpl36-c could functionally replace rpl36-p without any major steric interference or loss of intermolecular contacts. In addition, rpl36-c is highly conserved between the haptophyte and cryptophytes as would be expected for a functional ribosomal protein.

\section{Rubisco HGT revisited}

It was first recognized nearly 20 years ago [51-53] that red algae and their secondary symbiotic derivatives possess a rubisco operon $(r b c L S)$ of highly unusual evolutionary origin. Whereas all green plastids and those of glaucophytes contain $r b c L S$ of expected cyanobacterial origin, red plastids possess rubisco genes of apparent proteobacterial origin. Based on phylogenetic considerations, Delwiche and Palmer [16] argued in 1996 that the red algal 
rubisco was acquired from proteobacteria by horizontal transfer in the common ancestor of all red algae. In addition, they provided evidence for several other $r b c L S$ transfers, all among eubacteria. Martin and Schnarrenberger [53] argued that the cyanobacterial endosymbiont instead carried both the red-like and green-like rubisco genes from a duplication predating cyanobacteria and proteobacteria, and that differential loss in the plastid lineages and loss in all cyanobacterial lineages resulted in the observed pattern.

In the context of the current study, and with the passage of some 10 years and the accumulation of hundreds of bacterial genome sequences, we revisit this issue. Figure 4 shows an $r b c L$ phylogeny for a representative sampling of currently available sequences. The overall structure of this tree is very similar to that of Figure 2 of the paper by Delwiche and Palmer [16]. Importantly, however, a number of new proteobacterial $r b c L$ sequences have become available that show even greater similarity to red algal $r b c L$ than those considered by that study [16]. For example, the recently sequenced genome of Nitrosospira multiformis shares $86 \%$ amino-acid identity with Gracilaria over a contiguous 381 amino-acid region of $r b c L$. This is within the range of identities among the red algae over this same region. The $r b c L$ tree (Figure 4 ) groups the red algal $r b c L$ genes with those of Nitrosospira and Nitrosococcus. With the advent of these and other related bacterial sequences, the red algal $r b c L$ clade is now two steps nested within the overall clade of red-like proteobacterial $r b c L$ sequences, whereas previously [16] it was simply sister to a more limited set of proteobacteria.

Now, with many more rubisco sequences in hand, and with complete genomes available for many of these organisms, the duplication/loss model strongly conflicts with the phylogenetic and presence/absence data. None of the 15 or more sequenced cyanobacterial genomes contains a red-like $r b c L$. Furthermore, out of the many bacterial $r b c L$ sequences now available, only a single organism, Rhodobacter azotoformans, has been found that contains both red-like and green-like $r b c L$ [54], and this is clearly due to a bacterial HGT event instead of retention of both copies from an ancient duplication. So the hypothesis of an ancient duplication and differential loss of paralogs is becoming increasingly untenable.

Alternatively, instead of a transfer to the recent ancestor of the red algae, a recent ancestor of the cyanobacterial endosymbiont could have received a red-like $r b c L$ from a proteobacterium followed by differential losses in the plastid lineages [55]. This possibility is less parsimonious, however, as it still requires one horizontal transfer, plus at least two independent losses in the plastid lineage and at least one in cyanobacteria. In conclusion, it is likely that the $r b c L S$ operon of red algae represents a genuine HGT event to the plastid genome.

\section{HGT in plastids: rare but choice}

Comprehensive examination of all 204 genes present in four or more of the 42 examined plastid genomes has revealed but a single new, well-supported case of HGT. This $r p l 36$ transfer and the rbcLS transfer described years ago [16] and revisited above share several features: (i) they both involve bacterial donors; (ii) they are both relatively ancient [56-58], having occurred in the common ancestor of red algae ( $r b c L S)$, perhaps 1.0-1.5 billion years ago, or in the common ancestor of cryptophytes and haptophytes (rpl36), probably not much more recently; and (iii) in both cases, the transferred genes are known ( $r b c L S$; see Delwiche and Palmer [16,51-53], Valentin and Zetche [16,51-53], Boczar et al [16,51-53], Martin and Schnarrenberger [16,51-53], and references therein) or thought (rpl36) to have functionally replaced native homologs, which would explain their retention for eons as intact genes. The rpl36 transfer also serves as an important phylogenetic marker and is intriguing from a mechanistic standpoint. Thus, although HGT in plastids is extremely rare, when it happens it can be of considerable consequence and interest.

Both cases of plastid horizontal gene transfer occurred anciently in red algae or their secondary derivatives, while several cases of potential [22] or likely [20,21] horizontal acquisition of introns are evident in green algae plastids. In contrast, no cases of HGT were evident in our analyses of sequenced plastid genomes of land plants, nor has HGT been reported for any of the many plastid genes that have been widely sequenced (in hundreds to thousands of plants) for phylogenetic purposes. This contrast is noteworthy because far more plastid sequencing has been performed in land plants (99671 entries from an NCBI Entrez search for plastid genes) than in algae (6731 entries). Are algal plastids, an admittedly paraphyletic group, somehow more amenable to HGT than plant plastids?

\section{HGT and getting the right tree}

We initially constructed many phylogenetic trees that gave well-supported, conflicting results suggestive of HGT, but which were ultimately deemed wrong or showed weakened support under closer scrutiny. The largest source of conflicts arose within the red algal lineage and their secondary descendants (and to a lesser extent with the green algae), where limited taxon sampling and early diversification of lineages led to a series of long terminal branches connected by short internal branches. This is where phylogenetic reconstruction is most prone to fail due to LBA [59]. Subsequent analyses with improved taxon sampling and/or filtering of fast-evolving codon positions caused us 


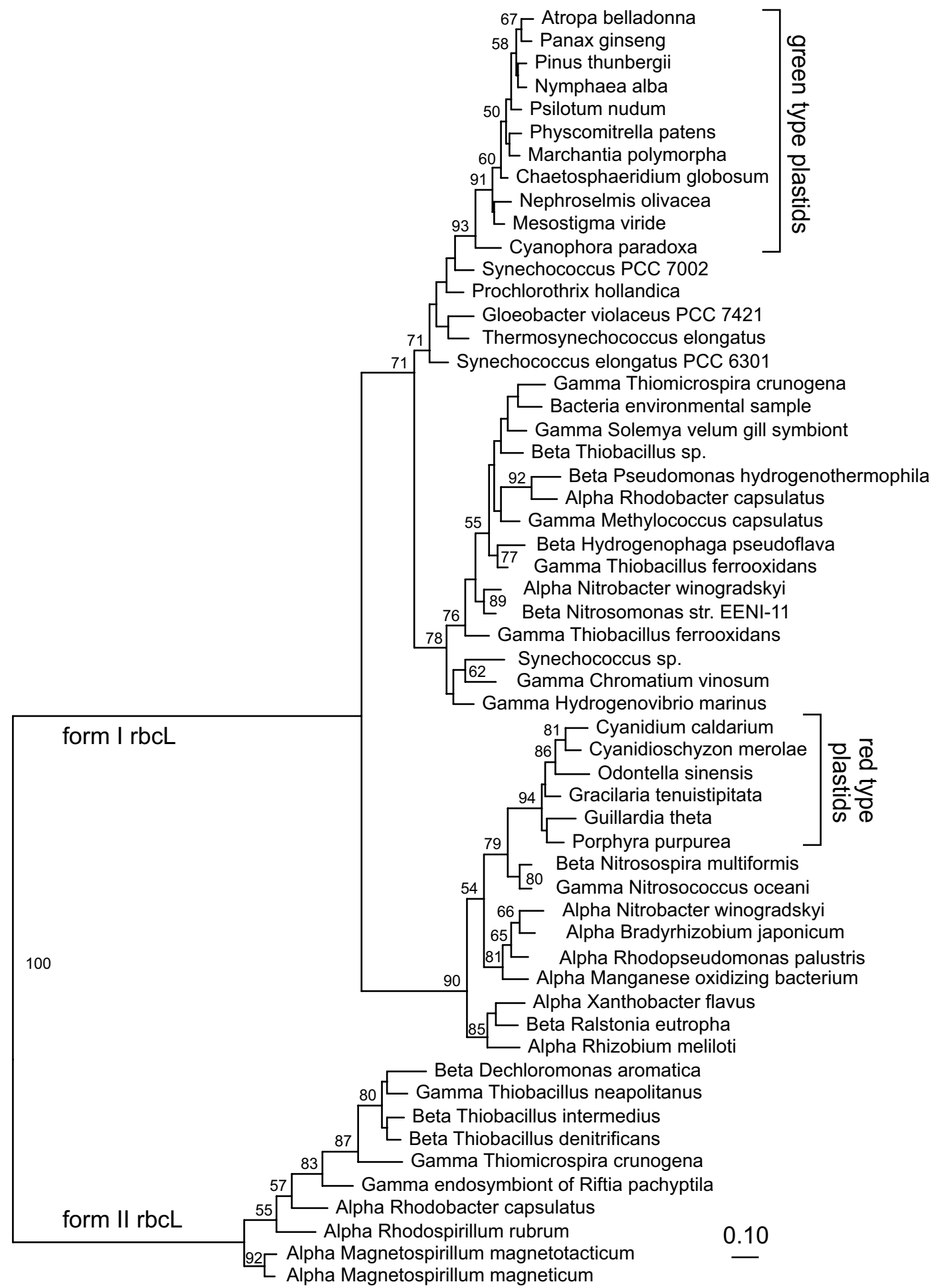

\section{Figure 4}

Phylogenetic tree of red-like and green-like $r b c L$ sequences. The amino-acid Bayesian tree was generated using MrBayes with the following parameters: rates = invgamma; aamodelpr = mixed; $n g e n=500000 ;$ nchains $=4$. The burnin was set to 100 to generate the tree and this burnin gave a convergence diagnostic of 0.017 . The nodal support values are PROML bootstrap support values obtained using global rearrangements, and four rate categories and an invariant category estimated using PUZZLE. Support values are shown on nodes with $B P \geq 50$. 
to reject all of these cases as potential HGTs. Some of these conflicts might still represent actual HGT events, but further taxon sampling will be required to resolve the issue completely. Sequencing errors should be considered a possibility and the anomalous sequences verified where appropriate.

\section{Why is HGT so less common in plastids than in mitochondria in land plants?}

Some 40 cases of HGT have now been reported in plant mitochondrial genomes [6-15] versus none in plant plastids. This is despite far less sequencing of plant mitochondrial genes (7075 Genbank entries) than plastid genes (99671 Genbank entries). Similarly, plant mitochondrial genomes are rich in plastid and nuclear sequences acquired via intracellular gene transfer [1-3], whereas plastid genomes entirely lack such sequences [4,5]. What could account for such differences? To be sure, plant mitochondrial genomes are less compact (72-89\% noncoding DNA in sequenced angiosperm genomes) and less constrained in size (varying over 10-fold in size). Nevertheless, angiosperm plastids contain considerable noncoding DNA (generally 40-45\%), suggesting that the greater propensity for mitochondrial HGT is not simply a function of the total amount of "junk" DNA. Rather, the differences may be how efficiently the organelles take up exogenous DNA. Plant mitochondria possess an active DNA uptake system [2]; no similar activity has been reported for plastids, but it is also unclear whether this has been assayed for. This uptake system may lower a rate-limiting barrier in the incorporation of both foreign and native DNA. A major, well-documented difference between the two organelles is the tendency of mitochondria to fuse. This may account for some of the observed mitochondrion-tomitochondrion HGT. Plant mitochondria regularly fuse $[60,61]$, promoting recombination between parental mitochondrial genomes in the case of somatic hybrid plants generated by protoplast fusion, whereas chloroplasts virtually never fuse under similar conditions $[62,63]$.

\section{Conclusion}

This study confirms and quantifies the hypothesis that HGT is rare in plastids. Only rpl36 and the rubisco operon are clear cases of HGT to the plastid genome. Both are ancient transfers, whereby bacterial genes have replaced native homologs and have become permanent, functional residents in their respective lineages. In contrast, the frequent (and recent) transfers in plant mitochondria occur by plant-to-plant transfer and are essentially ephemeral events, few of which seem to be of functional significance. The horizontal transfer of bacterial rpl36-c into the plastid genome represents an unprecedented example of apparent homologous recombination that defies current concepts of the sequence relatedness required to allow gene conversion/replacement to occur. The rpl36-c HGT also serves as a striking phylogenetic character that establishes an important new phylogenetic connection, linking haptophyte and cryptophyte plastids as sister groups to the exclusion of heterokont and alveolate plastids.

\section{Methods \\ Plastid genomes}

EMBL-Bank files for the following 40 plastid genomes were retrieved from the European Bioinformatics Institute: Eimeria tenella (Y217738), Euglena gracilis $(\underline{\mathrm{X} 70810})$, Euglena longa ( $\underline{\mathrm{AJ} 294725})$, Guillardia theta (AF041468), Toxoplasma gondii ( $\underline{\mathrm{U} 87145)}$, Cyanophora par-

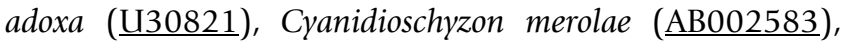
Cyanidium caldarium (AF022186), Gracilaria tenuistipitata

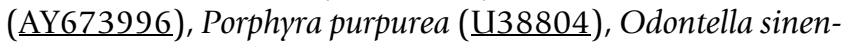

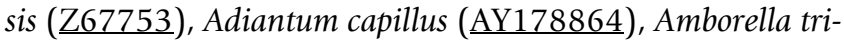
chopoda (J506156), Anthoceros formosae (스086179), Arabidopsis thaliana (AP000423), Atropa belladonna (AJ316582), Calycanthus floridus (AJ428413), Chaetosphae-

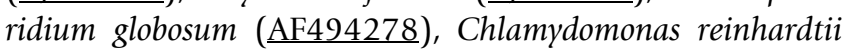
(BK000554), Chlorella vulgaris (스001684), Epifagus vir-

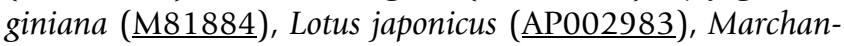
tia polymorpha (X04465), Medicago truncatula (AC093544), Mesostigma viride (AF166114), Nephroselmis olivacea (F137379), Nicotiana tabacum (Z00044), Nym-

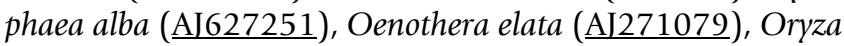
sativa (15901), Physcomitrella patens ( $\underline{\mathrm{AP005672}})$, Pinus koraiensis (AY228468), Pinus thunbergii (D17510), Psilotum nudum (AP004638), Saccharum officinarum (AP006714), Spinacia oleracea (ㅈ400848), Triticum aesti-

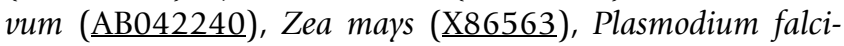
parum ( $\underline{\text { X95275 }}, \underline{\text { X95276 }})$, Panax ginseng (Y582139). In addition, the sequence of the Pisum sativum plastid genome was provided by John C. Gray (unpublished data), and the Vitis vinifera coding sequences were extracted and pieced together (unpublished result) from all NCBI nucleotide databases including dbEST [64]. A combination of BLAST searches with closely related genomes, Perl scripts for parsing output, and hand editing was used to define the protein and RNA genes in the unannotated genomes of Pisum sativum, Vitis vinifera, and Medicago truncatula.

\section{Gene clustering}

In total, 5676 protein, tRNA and rRNA genes were extracted from the 42 plastid genome sequences. A BLAST [65] database was created with these DNA sequences, and then each sequence was used as a BLAST query against the database. From the BLAST output, a pairwise distance matrix was constructed based on the best BLAST expectation value for each query/hit pair. For a pair to be considered, the BLAST expectation value had to be $\leq 0.1$, and at least $20 \%$ of the longer sequence of a pair had to be included in the alignment. Pairs for which these criteria 
were not met received a large distance value of 1.1. A huge neighbor-joining tree was constructed with PAUP* software, [66] using this distance matrix. Gene families and superfamilies were easily identifiable in the resulting tree. Unrelated gene families formed a polytomy of long branches at the root node. From this tree, 204 protein gene families containing four or more genes were hand selected by visual inspection. Ribosomal RNA genes were easily resolved in the tree, but transfer RNA genes were clustered into many hard-to-resolve paralogous families. The distinct tRNA clusters were separated into groups for further clustering using maximum parsimony (MP) and ML analyses.

\section{Gene alignment}

Protein and nucleotide alignments were made for each of the gene families using MUSCLE software [67] with unlimited iterations, and were inspected manually to correct errors. Initially, amino-acid alignments were constructed for sequences whose translation could be obtained. The protein alignment was then back-translated to nucleotides using the known nucleotide sequences. Sequences that could not be translated (such as pseudogenes and RNA genes) were aligned based on nucleotides. Positions containing mostly gaps, especially where homology was deemed questionable, were excluded from phylogenetic analyses.

\section{Phylogenetic analyses}

ML models for each gene family were determined using the likelihood-ratio test criterion of MODELTEST [68] except where specified. Final model parameter values were estimated by iteratively building ML trees and recalculating parameter values until the best trees converged. Heuristic ML searches using tree bisection-reconnection branch swapping in PAUP* were performed to find these trees. All three codon positions were included for the initial phylogenetic screening of gene families, but first and second position and other character-sampling strategies and software were used secondarily if needed to clarify the phylogenetic support for a conflicting tree. One hundred ML bootstrap replicates were performed using the same model and search method as used for searches for the best trees. Neighbor-joining and MP analyses were also carried out to allow for comparison to the ML results. Additional phylogenetic methods and programs (e.g. MrBayes, PAML and PROML) are indicated where used.

\section{Phylogenetic conflict evaluation}

A consensus plastid tree (Additional File 1) was used as the working hypothesis topology for finding conflicts in gene trees. This tree was compiled from the current literature and our unpublished work using entire genomes. Nodes in a gene tree that conflicted with the plastid tree by addition of a taxon not part of that clade or subtraction of a taxon that is part of that clade were marked as in conflict. Conflicting nodes were ordered by their ML BP values, using a PERL script developed for finding these conflicts. Trees were viewed graphically, with conflicting clades highlighted to determine whether further processing was necessary. To rule out HGT in well-supported conflicts, we scrutinized the alignment in more detail, increased taxon sampling, and tried other models and phylogenetic methods.

\section{New gene sequences}

Several new sequences for rpl36 (DQ365944DQ365946) and $y c f 2$ (DQ370441-DQ370447) were obtained using standard PCR and sequencing protocols [69]. Cryptophyte genomic DNAs (for rpl36 isolation) were generously provided by Chris Lane and John Archibald. Angiosperm DNAs (for $y c f 2$ isolation) were isolated [70] directly from young plant leaves or were taken from lab stocks.

\section{Authors' contributions}

DWR carried out all the analyses, wrote the new software used in this study, generated the new rpl36 and $y c f 2$ sequence data, and drafted the manuscript (text and figures). DWR and JDP jointly directed the project, and JDP contributed substantially to the final manuscript. Both authors read and approved the final manuscript.

\section{Additional material}

\section{Additional file 1}

Consensus plastid phylogeny. Shown is the plastid phylogeny used in this study, based on the current literature. Dashed and solid vertical brackets denote paraphyletic and monophyletic groups, respectively. Although the tree is shown as entirely resolved, some parts are not well supported (e.g., relationships among Cyanophora, green algae, and red algae; among bryophytes; among chromalveolates; and whether Amborella plus Nymphaea or Amborella alone is the sister of all other angiosperms). Click here for file

[http://www.biomedcentral.com/content/supplementary/17417007-4-31-S1.pdf] 


\section{Additional file 2 \\ Amino-acid alignment of secY, rpl36 and $\mathrm{rps} 13$ and the rpl36 amino- acid alignment showing the $C$-terminal extensions and atomic contacts in the ribosome crystal structure. (A) This shows that $\mathrm{secY}$ and $\mathrm{rps} 13$ from plastids which contain the c-type $\mathrm{rpl} 36$ are more similar to secY and rps13 genes from red algae and cyanobacteria than to potential rpl36 donors, Rhodopirellula and proteobacteria. Amino acids that conflict with the consensus amino acid at each position are colored according to the key. The similarity between the c-type rpl36 genes in the haptophyte and cryptophyte plastids and those from Rhodopirellula and proteobac- teria is also apparent. Note that rpl36 is flanked by secY and rps13 in plastids of red algal origin, but the c-type rpl36 in bacteria is not flanked by these genes. (B) Amino-acid alignment of c-type and p-type rpl36 genes. Note the three apicomplexans included in the alignment (Plasmo- dium, Toxoplasma, and Theileria). At the bottom is shown which resi- dues make contact with the $23 S \mathrm{RNA}$ in the ribosome crystal structure of Escherichia coli, which has a p-type rpl36. \\ Click here for file \\ [http://www.biomedcentral.com/content/supplementary/1741- 7007-4-31-S2.pdf]}

\section{Additional file 3}

The poorly resolved rpl36 tree obtained in the initial phylogenetic screen. This shows the Guillardia rpl36 gene going arbitrarily with Pisum with weak support given the poor taxon sampling and low information content of this gene. See Figure 1 for clarification.

Click here for file

[http://www.biomedcentral.com/content/supplementary/17417007-4-31-S3.pdf]

\section{Additional file 4}

Discussion of the Oenothera elata ycf2 gene error in the genome sequence. Discussion of the Oenothera elata ycf2 gene error in the genome sequence.

Click here for file

[http://www.biomedcentral.com/content/supplementary/17417007-4-31-S4.pdf]

\section{Additional file 5}

Results of the psbA conflict in the red algal lineage. Results of the psbA conflict in the red algal lineage.

Click here for file

[http://www.biomedcentral.com/content/supplementary/17417007-4-31-S5.pdf]

\section{Additional file 6}

Resolution of conflicts within grasses by adding taxa for the atpI gene. (A) The topology and support values with the original taxon sampling. The conflicting node is indicated by the bold 96; (B) after more monocot taxa are added Triticum and Zea move apart.

Click here for file

[http://www.biomedcentral.com/content/supplementary/17417007-4-31-S6.pdf]

\section{Additional file 7}

List of gene trees having branches $\geq 4 S D$ above the mean branch length for a given tree and text describing analysis of long branch taxa. The text describes the analysis of long-branch taxa. The table gives the number of standard deviations above the mean, the corresponding gene and the group that the long-branch leads to.

Click here for file

[http://www.biomedcentral.com/content/supplementary/1741-

7007-4-31-S7.pdf]

\section{Additional file 8}

Discussion of chromalveolate hypothesis and algal phylogeny. Discussion of chromalveolate hypothesis and algal phylogeny.

Click here for file

[http://www.biomedcentral.com/content/supplementary/17417007-4-31-S8.pdf]

\section{Additional file 9}

Multigene chromist plastid phylogeny. Results of the phylogenetic placement analysis of the haptophyte Emiliania based on all plastid genes. Click here for file

[http://www.biomedcentral.com/content/supplementary/17417007-4-31-S9.pdf]

\section{Additional file 10}

Plastid genes supporting the sisterhood of either haptophytes and heterokonts or haptophytes and cryptophytes. The histograms list the genes favoring the given topology over the other according to the codeml aminoacid ML score with the WAG matrix [71] and gamma distributed rates. The site-specific likelihood values were calculated using parameter estimates and branch lengths based on the concatenated alignment with all the genes. The height of the histogram bars represent the log likelihood preference for the given topology over the other for a particular gene (i.e. the sum of site likelihoods in the concatenated alignment corresponding to a particular gene). (A) The topology found using MrBayes with 21,659 plastid amino-acid positions shared among the chromophytes, Gracilaria, and Porphyra. Invariant sites and gamma distributed rates were used with the Cprev model [72]. The posterior probabilities were 1.0 for all nodes. (B) The topology was found as in (A) except that Guillardia and Emiliania were constrained to be monophyletic. The node corresponding to the chromist clade had a posterior probability of 0.98 and the other nodes had 1.0.

Click here for file

[http://www.biomedcentral.com/content/supplementary/17417007-4-31-S10.pdf]

\section{Acknowledgements}

We thank Christa Long and Les Goertzen for contributing unpublished clpP sequences for use in this study; Chris Lane, Hameed Khan, and John Archibald for providing several cryptophyte DNA samples and unpublished information about the Rhodomonas plastid genome; John Gray for providing the unpublished sequence of Pisum sativum plastid genome; and Virginia Sanchez-Puerta for helpful suggestions regarding the manuscript. This work was supported by NIH research grants GM-35087 and GM-706I2 (to J.D.P.).

\section{References}

I. Burger G, Gray MW, Lang BF: Mitochondrial genomes: anything goes. Trends Genet 2003, 19:709-7I6. 
2. Koulintchenko M, Konstantinov $\mathrm{Y}$, Dietrich A: Plant mitochondria actively import DNA via the permeability transition pore complex. EMBO J 2003, 22: | 245-I254.

3. Knoop V: The mitochondrial DNA of land plants: peculiarities in phylogenetic perspective. Curr Genet 2004, 46: I 23-139.

4. Palmer JD: Contrasting modes and tempos of genome evolution in land plantorganelles. Trends Genet 1990, 6: I I5-120.

5. Lemieux C, Otis C, Turmel M: Ancestral chloroplast genome in Mesostigma viride reveals an early branch of green plant evolution. Nature 2000, 403:649-652.

6. Cho Y, Qiu YL, Kuhlman P, Palmer JD: Explosive invasion of plant mitochondria by a group I intron. Proc Natl Acad Sci USA 1998, 95: |4244-|4249.

7. Cho Y, Palmer JD: Multiple acquisitions via horizontal transfer of a group I intron in the mitochondrial coxI gene during evolution of the Araceae family. Mol Biol Evol 1999, I 6: I 155-I 165.

8. Bergthorsson U, Adams KL, Thomason B, Palmer JD: Widespread horizontal transfer of mitochondrial genes in flowering plants. Nature 2003, 424:197-201.

9. Won $\mathrm{H}$, Renner SS: Horizontal gene transfer from flowering plants to Gnetum. Proc Natl Acad Sci USA 2003, I 00: I 0824-I0829.

10. Nickrent DL, Blarer A, Qiu YL, Vidal-Russell R, Anderson FE: Phylogenetic inference in Rafflesiales: the influence of rate heterogeneity and horizontal gene transfer. BMC Evol Biol 2004, 4:40.

II. Mower JP, Stefanovic S, Young G], Palmer JD: Plant genetics: gene transfer from parasitic to host plants. Nature 2004, 432:165-166.

12. Davis CC, Wurdack KJ: Host-to-parasite gene transfer in flowering plants: phylogenetic evidence from Malpighiales. Science 2004, 305:676-678.

13. Bergthorsson U, Richardson AO, Young GJ, Goertzen LR, Palmer JD: Massive horizontal transfer of mitochondrial genes from diverse land plant donors to the basal angiosperm Amborella. Proc Natl Acad Sci USA 2004, I 0 I : I7747- 17752.

14. Davis CC, Anderson WR, Wurdack KJ: Gene transfer from a parasitic flowering plant to a fern. Proc Biol Sci 2005, 272:2237-2242.

15. Schonenberger J, Anderberg AA, Sytsma KJ: Molecular phylogenetics and patterns of floral evolution in the Ericales. Int Plant Sci 2005, 166:265-288.

16. Delwiche CF, Palmer JD: Rampant horizontal transfer and duplication of Rubisco genes in eubacteria and plastids. Mol Biol Evol 1996, 3 I:873-882.

17. Turmel M, Gutell RR, Mercier JP, Otis C, Lemieux C: Analysis of the chloroplast large subunit ribosomal RNA gene from 17 Chlamydomonas taxa. Three internal transcribed spacers and I 2 group I intron insertion sites. J Mol Biol I993, 232:446-467.

18. Maier UG, Rensing SA, Igloi GL, Maerz M: Twintrons are not unique to the Euglena chloroplast genome - structure and evolution of a plastome cpn60 gene from a cryptomonad. Mol Gen Genet 1995, 246: I28-I3I.

19. Nozaki H, Ohta N, Yamada T, Takano H: Characterization of rbcL group IA introns from two colonial volvocalean species (Chlorophyceae). Plant Mol Biol 1998, 37:77-85.

20. Sheveleva EV, Hallick RB: Recent horizontal intron transfer to a chloroplast genome. Nucleic Acids Res 2004, 32:803-810.

21. Odom OW, Shenkenberg DL, Garcia JA, Herrin DL: A horizontally acquired group II intron in the chloroplast psbA gene of a psychrophilic Chlamydomonas: in vitro self-splicing and genetic evidence for maturase activity. RNA 2004, 10:1097-I 107

22. Thompson MD, Copertino DW, Thompson E, Favreau MR, Hallick $\mathrm{RB}$ : Evidence for the late origin of introns in chloroplast genes from an evolutionary analysis of the genus Euglena. Nucleic Acids Res 1995, 23:4745-4752.

23. Doetsch NA, Thompson MD, Favreau MR, Hallick RB: Comparison of psbK operon organization and group III intron content in chloroplast genomes of I 2 Euglenoid species. Mol Gen Genet 200I, 264:682-690.

24. Hanyuda T, Arai S, Ueda K: Variability in the rbcL introns of Caulerpalean algae (Chlorophyta, Ulvophyceae). I Plant Res 2000, I I 3:403-4 I3

25. Pombert JF, Otis C, Lemieux C, Turmel M: Chloroplast genome sequence of the green alga Pseudendoclonium akinetum (Ulvophyceae) reveals unusual structural features and new insights into the branching order of chlorophyte lineages. Mol Biol Evol 2005, 22:1903-1918.
26. Hoef-Emden K, Marin B, Melkonian M: Nuclear and nucleomorph SSU rDNA phylogeny in the Cryptophyta and the evolution of cryptophyte diversity. J Mol Evol 2002, 55:16I-179.

27. Sanchez-Puerta MV, Bachvaroff TR, Delwiche CF: The complete plastid genome sequence of the haptophyte Emiliania huxleyi: a comparison to other plastid genomes. DNA Res 2005, I 2: $|5|-\mid 56$

28. Ishida K, Green BR: Second- and third-hand chloroplasts in dinoflagellates: phylogeny of oxygen-evolving enhancer I (PsbO) protein reveals replacement of a nuclear-encoded plastid gene by that of a haptophyte tertiary endosymbiont. Proc Natl Acad Sci USA 2002, 99:9294-9299.

29. Yoon HS, Hackett JD, Van Dolah FM, Nosenko T, Lidie KL, Bhattacharya $D$ : Tertiary endosymbiosis driven genome evolution in dinoflagellate algae. Mol Biol Evol 2005, 22:1299-I308.

30. Patron NJ, Waller RF, Keeling PJ: A tertiary plastid uses genes from twoendosymbionts. I Mol Biol 2006, 357:1373-1382

31. Hupfer H, Swiatek M, Hornung S, Herrmann RG, Maier RM, Chiu WL, Sears B: Complete nucleotide sequence of the Oenothera elata plastid chromosome, representing plastome I of the five distinguishable euoenothera plastomes. Mol Gen Genet 2000, 263:58I-585

32. Keeling PJ: Diversity and evolutionary history of plastids and their hosts. Am J Bot 2004, 9 I:|48|-|493.

33. Inagaki Y, Simpson AGB, Dacks JB, Roger AJ: Phylogenetic artifacts can be caused by leucine, serine, and arginine codon usage heterogeneity: Dinoflagellate plastid origins as a case study. Syst Biol 2004, 53:582-593.

34. Stefanovic S, Rice DW, Palmer JD: Long branch attraction, taxon sampling, and the earliest angiosperms: Amborella or monocots? BMC Evol Biol 2004, 4:35.

35. Turmel M, Ehara M, Otis C, Lemieux C: Phylogenetic relationships among streptophytes as inferred from chloroplast small and large subunit rRNA gene sequences. J Phycol 2002 , 38:364-375

36. Cavalier-Smith T: Origin and relationships of Haptophyta. In The Haptophyte algae Edited by: Green JC, Leadbeater BSC. Oxford: Clarendon Press; 1994:413-435.

37. Cavalier-Smith T: Genomic reduction and evolution of novel genetic membranes and protein-targeting machinery in eukaryote-eukaryote chimaeras (meta-algae). Philos Trans $R$ Soc Lond B Biol Sci 2003, 358: 109-133. discussion I 33-140

38. Bachvaroff TR, Sanchez-Puerta MV, Delwiche CF: Chlorophyll ccontaining plastid relationships based on analyses of a multigene data set with all four chromalveolate lineages. Mol Biol Evol 2005, 22: I772-I782.

39. Yoon HS, Hackett JD, Pinto G, Bhattacharya D: The single, ancient originof chromist plastids. Proc Natl Acad Sci USA 2002, 99:15507-15512

40. Cavalier-Smith T: Principles of protein and lipid targeting in secondarysymbiogenesis: Euglenoid, dinoflagellate, and sporozoan plastid origins and the eukaryote family tree. J Eukaryot Microbiol 1999, 46:347-366.

41. Harper JT, Waanders E, Keeling PJ: On the monophyly of chromalveolates using a six-protein phylogeny of eukaryotes. Int I Syst Evol Microbiol 2005, 55:487-496.

42. Majewski J, Cohan FM: DNA sequence similarity requirements for interspecific recombination in Bacillus. Genetics 1999 , I53:|525-1533

43. Thomas CM, Nielsen KM: Mechanisms of, and barriers to, horizontal gene transfer between bacteria. Nat Rev Microbiol 2005 , 3:7||-72।.

44. Boucher Y, Douady CJ, Sharma AK, Kamekura M, Doolittle WF: Intragenomic heterogeneity and intergenomic recombination among haloarchaeal rRNA genes. J Bacteriol 2004, I 86:3980-3990.

45. Inagaki Y, Susko E, Roger AJ: Recombination between elongation factor I-alpha genes from distantly-related archaeal lineages. Proc Natl Acad Sci USA 2006 in press.

46. Ikeda $\mathrm{H}$, Shiraishi $\mathrm{K}$, Ogata $\mathrm{Y}$ : Illegitimate recombination mediated by double-strand break and end-joining in Escherichia coli. Adv Biophys 2004, 38:3-20.

47. Cohan FM: Bacterial species and speciation. Syst Biol 200I, 50:513-524.

48. Bajaj M, Blundell T: Evolution and the tertiary structure of proteins. Annu Rev Biophys Bioeng 1984, 13:453-492. 
49. Knight RD, Freeland SJ, Landweber LF: A simple model based on mutation and selection explains trends in codon and aminoacid usage and GC composition within and across genomes. Genome Biol 200I, 2:RESEARCHOOIO.

50. Schuwirth BS, Borovinskaya MA, Hau CW, Zhang W, Vila-Sanjurjo A, Holton JM, Cate JH: Structures of the bacterial ribosome at 3.5 A resolution. Science 2005, 3 1 0:827-834.

5I. Valentin K, Zetsche K: The genes of both subunits of ribuloseI,5-bisphosphate carboxylase constitute an operon on the plastome of a red alga. Curr Genet 1989, 16:203-209.

52. Boczar BA, Delaney TP, Cattolico RA: Gene for the ribulose-I,5bisphosphate carboxylase small subunit protein of the marine chromophyte Olisthodiscus luteus is similar to that of a chemoautotrophic bacterium. Proc Natl Acad Sci USA 1989, 86:4996-4999.

53. Martin W, Schnarrenberger C: The evolution of the Calvin cycle from prokaryotic to eukaryotic chromosomes: a case study of functional redundancy in ancient pathways through endosymbiosis. Curr Genet 1997, 32: I-I8.

54. Úchino Y, Yokota A: "Green-like" and "red-like" RubisCO cbbL genes in Rhodobacter azotoformans. Mol Biol Evol 2003, 20:82I-830.

55. Assali NE, Martin WF, Sommerville CC, Loiseaux-de Goër S: Evolution of the Rubisco operon from prokaryotes to algae: Structure and analysis of the rbcS gene of the brown alga Pylaiella littoralis. Plant Mol Biol I991, I 7:853-863.

56. Douzery EJP, Snell EA, Bapteste E, Delsuc F, Philippe H: The timing of eukaryotic evolution: Does a relaxed molecular clock reconcile proteins and fossils? Proc Natl Acad Sci USA 2004, I 0 I:I5386-15391.

57. Yoon HS, Hackett JD, Ciniglia C, Pinto G, Bhattacharya D: A molecular timeline for the origin of photosynthetic eukaryotes. Mol Biol Evol 2004, 2 1:809-818.

58. Butterfield NJ: Bangiomorpha pubescens n. gen., n. sp.: implications for the evolution of sex, multicellularity, and the Mesoproterozoic/Neoproterozoic radiation of eukaryotes. Paleobiology 2000, 26:386-404.

59. Schulmeister S: Inconsistency of maximum parsimony revisited. Syst Biol 2004, 53:521-528.

60. Arimura S, Yamamoto J, Aida GP, Nakazono M, Tsutsumi N: Frequent fusion and fission of plant mitochondria with unequal nucleoid distribution. Proc Natl Acad Sci USA 2004, | 0 I:7805-7808.

6I. Sheahan MB, McCurdy DW, Rose RJ: Mitochondria as a connected population:ensuring continuity of the mitochondrial genome during plant cell dedifferentiation through massive mitochondrial fusion. Plant J 2005, 44:744-755.

62. Mohapatra T, Kirti PB, Kumar VD, Prakash S, Chopra VL: Random chloroplast segregation and mitochondrial genome recombination in somatic: hybrid plants of Diplotaxis catholica + Brassica juncea. Plant Cell Rep 1998, 17:814-818.

63. Kanno A, Kanzaki H, Kameya T: Detailed analyses of chloroplast and mitochondrial DNAs from the hybrid plant generated by asymmetric protoplast fusion between radish and cabbage. Plant Cell Rep 1997, 16:479-484.

64. Boguski MS, Lowe TM, Tolstoshev CM: dbEST - database for "expressed sequence tags". Nat Genet 1993, 4:332-333.

65. Altschul SF, Madden TL, Schaffer AA, Zhang J, Zhang Z, Miller W, Lipman DJ: Gapped BLAST and PSI-BLAST: a new generation of protein database search programs. Nucleic Acids Res 1997, 25:3389-3402.

66. Swofford DL: PAUP*: Phylogenetic analysis using parsimony (* and other methods). Version 4.0b 10 Sunderland, Massachusetts: Sinauer Associates; 2003

67. Edgar RC: MUSCLE: multiple sequence alignment with high accuracy and high throughput. Nucleic Acids Res 2004 , 32:1792-1797.

68. Posada D, Crandall KA: MODELTEST: testing the model of DNA substitution. Bioinformatics 1998, 14:817-818.

69. Cho Y, Mower JP, Qiu YL, Palmer JD: Mitochondrial substitution rates are extraordinarily elevated and variable in a genus of flowering plants. Proc Natl Acad Sci USA 2004, I 0 I : | 774 |- I 7746.

70. Doyle J], Doyle JL: A rapid DNA isolation procedure for small quantitiesof fresh leaf tissue. Phytochem Bull 1987, I9: I I-I5.
7I. Whelan S, Goldman N: A general empirical model of protein evolution derived from multiple protein families using a maximum-likelihood approach. Mol Biol Evol 200I, I 8:69|-699.

72. Adachi J, Waddell PJ, Martin W, Hasegawa M: Plastid genome phylogeny anda model of amino acid substitution for proteins encoded by chloroplast DNA. J Mol Evol 2000, 50:348-358.
Publish with Biomed Central and every scientist can read your work free of charge

"BioMed Central will be the most significant development for disseminating the results of biomedical research in our lifetime. "

Sir Paul Nurse, Cancer Research UK

Your research papers will be:

- available free of charge to the entire biomedical community

- peer reviewed and published immediately upon acceptance

- cited in PubMed and archived on PubMed Central

- yours - you keep the copyright

Submit your manuscript here:

http://www.biomedcentral.com/info/publishing_adv.asp
BioMedcentral 